\title{
Prevalencia Serológica de Salmonella enteritidis en la Población Canina del Municipio de Tunja, Colombia
}

\author{
The serological prevalence of Salmonella enteritidis amongst \\ Tunja's canine population (a large Colombian city)
}

\author{
Oscar Rincón-Reyes y Judith Figueroa
}

Departamento de Ciencias para la Salud Animal,Universidad Nacional de Colombia. Bogotá. E-mail: oscarrinconvet@yahoo.es, jfigueroaa@unal.edu.co.

Recibido 29 Agosto 2007/Enviado para Modificación 19 Abril 2008/Aceptado 4 Junio 2008

\section{RESUMEN}

Objetivo Determinar la prevalencia serológica de Salmonella enteritidis en la población canina de Tunja (Boyacá), durante julio y octubre del 2006 y establecer la relación entre los factores predisponentes y la serorreactividad en la población canina.

Materiales y Métodos Sobre la población canina estimada en 9623 animales (censo de 2002), y asumiendo una prevalencia critica preestablecida de $3 \%$ y un nivel de confianza del $95 \%$, se recolectaron 72 muestras sanguíneas caninas distribuidas en cinco zonas de la ciudad, se aplicó una encuesta para cada animal para determinar la presencia de factores predisponentes y un examen clínico completo. Con los sueros obtenidos, se tituló el nivel de anticuerpos mediante la prueba de microaglutinación en placa para Salmonella enteritidis (MAG).

Resultados La prevalencia serológica a Salmonella enteritidis fue del 41,7\%, las prevalencias zonales fueron: $10 \%$ Norte, $10 \%$ occidente, $16,7 \%$ Oriente, 36,7 \% sur y $26,6 \%$ centro. Se determinó una correlación directa con la presentación de problemas entéricos anteriores $(p<0,05)(O R 3,5)$ y en menor grado con el acceso a desechos orgánicos $(p<0,05)(O R 0,4)$; otros factores como la convivencia con otros animales y la salud mostraron niveles de riesgo positivo (OR 1,4 Y OR 2).

Conclusiones Existe una alta prevalencia serológica a Salmonella enteritidis en la ciudad de Tunja, con mayor incidencia en la zona sur, siendo el principal factor predisponente la presentación de problemas entéricos anteriores, el cual es un factor de alto riesgo en la diseminación de esta entidad.

Palabras Clave: Salmonella enteritidis, prevalencia, zoonosis (fuente: DeCS, BIREME).

\section{ABSTRACT}

Objective Determining the serological prevalence of Salmonella enteritidis in the city of Tunja's canine population (in Boyacá) from July to October 2006 and establishing the relationship between predisposed factors and seroreactivity within this canine population. Materials and Methods 72 canine blood samples were gathered from all of the 
city's five areas from an estimated 9623 canine population (2002 census), assuming a $3 \%$ pre-established critical prevalence and $95 \%$ confidence level. A survey was made of each animal to determine the presence of predisposed factors and they were all given a complete clinical examination. The sera so obtained were used for titering antibody levels by means of the microagglutination plate test for Salmonella enteritidis (MAG).

Results Salmonella enteritidis serological prevalence was 41,7\%. Area prevalence within the city was $10 \%$ for the north, $10 \%$ for the west, $16,7 \%$ for the east, 36,7 \% the south and $26,6 \%$ for the city-center. A direct correlation was established with the presentation of previous enteric problems $(p<0.05)(O R$ 3.5) and, to a lesser extent, with access to organic waste $(p<0.05)(O R 0.4)$. Other factors presented positive risk levels, such as coexistence with other animals and health (OR 1.4 and OR 2)

Conclusions Salmonella enteritidis had a high serological prevalence in the city of Tunja, most incidents occurring in the southern area. The presentation of previous enteric problems was the main predisposed factor, this being a high risk factor in this entity's dissemination.

Key Words: Salmonella enteritidis, prevalence, zoonoses (source: MeSH, NLM).

L a Salmonellosis es una enfermedad zoonótica (1). Los reservorios animales de esta enfermedad son variados, aves, animales de granja, perros, gatos, además de especies salvajes incluidos los reptiles (2) La enfermedad es causada por el género Salmonella del cual hay muchas especies que pueden afectar a los seres humanos. La enfermedad es de distribución mundial, siendo la Salmonella typhimurium uno de los serotipos más prevalentes en el mundo. Se han descrito más de 2000 serotipos de Salmonella (3).

En caninos el número de serotipos es amplio. Galton y Colaboradores pudieron recobrar 53 serotipos de caninos, demostrando además que estos serotipos suelen ser los mismos reportados en los humanos . En Estados Unidos, Moran identificó 23 serotipos caninos diferentes.

Algunos estudios de prevalencia determinan un potencial zoonótico de 2,3 \% para la Salmonella en caninos $(4,5)$, siendo claramente una de las fuentes comunes de infección por Salmonella en humanos (6); Las especies más comúnmente aisladas de caninos son la Salmonella typhimurium y enteritidis. Las fuentes más comunes son el contacto con alimentos contaminados, agua o fómites, debido a que Salmonella puede sobrevivir por periodos relativamente largos fuera del huésped (7); los alimentos no cocidos en la dieta han sido frecuentemente la causa de la infección, sin embargo, la Salmonella puede contaminar y replicarse en alimentos procesados durante el almacenamiento (8). El estrés o una terapia inmunosupresora, así como enfermedades concurrentes 
Prevalencia Salmonella canina

y sobrepoblación aumentan el riesgo de contagio (9). La existencia de portadores asintomáticos humanos y animales y la resistencia de la Salmonella a los antibióticos, son factores que tienen gran significancia epidemiológica. $(3,4,10)$. Se estima que Salmonella spp. infecta 1,4 millones de personas anualmente en Estados Unidos, aunque muchas infecciones son auto limitantes; las infecciones severas no son muy comunes (11). En promedio 15000 personas son hospitalizadas y más de 500 mueren anualmente (12). La transmisión a humanos, ocurre por ingestión de carne, productos lácteos y otros alimentos contaminados por heces de animales y por transmisión cruzada de alimentos contaminados por Salmonella $(13,14)$.

La transmisión zoonótica puede ocurrir a través de exposición directa a heces animales. Los caninos son una especie bastante prolífica la cual puede en 10 años crecer un $85 \%$ comparado con el 23,5\% de crecimiento en la población humana (15). La presencia de animales callejeros y la sobreoferta de esta especie favorecen la zoonosis.

\section{MATERIALES Y MÉTODOS}

El presente estudio fue realizado en la población canina de la ciudad de Tunja, en el año 2006, que alcanzó los 9623 animales (Censo de vacunación antirrábica, 2002).

El tamaño de la muestra se calculó mediante el empleo del programa Winepiscope (OMS-OPS. 2000) se asumió una prevalencia crítica preestablecida del $3 \%$, un nivel de confianza del $95 \%$, determinándose un mínimo estadístico de 72 muestras.

Las muestras se tomaron, teniendo en cuenta los diferentes puestos de vacunación y el área abarcada por los mismos; para lo cual la ciudad de dividió en 5 zonas.

Se seleccionaron al azar los hogares visitados 14 o 15 según el tamaño de la zona, luego se verificó la existencia de caninos y posteriormente con autorización de los propietarios se procedió a tomar muestras sanguíneas de cada animal (16), a su vez se diligenció una encuesta que incluyó los siguientes datos: zona, número, fecha, edad, sexo, raza, tipo de dieta, hábitat y condiciones higiénicas, acceso a desechos orgánicos, convivencia con otros animales, problemas entéricos anteriores, desparasitación, fuentes de agua, manejo de excretas, y por último se realizó un examen clínico completo al animal. 
A los sueros obtenidos se le aplicó la prueba de microaglutinación en placa (MAG) (17), utilizando un antígeno comercial de Salmonella enteritidis, diluido 1:10 en solución salina fenicada $0,5 \%$, los sueros fueron diluidos $1: 2$ en forma seriada desde 1:5 hasta $1: 10240$ y fueron enfrentados al antígeno a $37^{\circ} \mathrm{C}$ por 24 Horas, para la lectura del titulo de anticuerpos. Se empleó en la prueba un suero control positivo, que correspondió al suero de un canino diagnosticado previamente como negativo e inoculado con Salmonella enteritidis inactivada con formaldehído a una concentración de $10^{7} \mathrm{UFC} / \mathrm{ml}$. El animal fue inoculado con $1 \mathrm{ml} \mathrm{IM}$, semanalmente durante un mes, treinta días después de la última inoculación se efectuó la toma de las muestras para la obtención del suero. El control negativo se obtuvo de animales que no reaccionaron al antígeno durante la prueba. Una reacción de aglutinación en dilución 1:40 ó mayor se consideró positiva en la prueba de MAG (17).

El análisis estadístico fue de tipo descriptivo. Los datos obtenidos fueron organizados en tablas electrónicas en el programa Excel (Microsoft) y luego fueron analizados en EPIINFO 2002, utilizando como prueba estadística Chí2, calculando un límite de confianza del $95 \%$, considerando resultados estadísticamente significativos si $\mathrm{p}<0,05$

\section{RESULTADOS}

Los sueros de los caninos mostraron un 41,7 \% de reactores seropositivos y un $58,3 \%$ seronegativos. Los animales positivos en la ciudad indicaron prevalencias de $10 \%$ para la zona Norte, $10 \%$ para la zona Occidente, 16,7 \% para la zona Oriental, $36,7 \%$ para la zona sur y un $26,6 \%$ para la zona Centro (Tabla 1).

Tabla 1. Perros y positividad

\begin{tabular}{lccccc}
\hline \multirow{2}{*}{ Zona } & \multicolumn{2}{c}{ Perros } & \multirow{2}{*}{ Muestra } & \multicolumn{2}{c}{ Positividad } \\
\cline { 2 - 3 } \cline { 5 - 6 } & Número & $\%$ & & Número & $\%$ muestra \\
\hline Sur & 2130 & 22,1 & 15 & 11 & 73,3 \\
Oriente & 1969 & 20,5 & 15 & 5 & 33,3 \\
Oocidente & 3486 & 36,2 & 14 & 3 & 21,4 \\
Centro & 510 & 5,2 & 14 & 8 & 57,1 \\
Norte & 1530 & 15,9 & 14 & 3 & 21,4 \\
\hline Total & 9623 & 100 & 72 & 30 & 100
\end{tabular}

Los títulos de anticuerpos o positivos oscilaron entre 1:40 y 1: 10240 con un promedio de 1:2 271 y una desviación estándar de 3,2.

En cuanto a la asociación con los factores predisponentes, se encontró una relación entre los animales positivos y la presentación de problemas entéricos anteriores, $(p<0,05)(\mathrm{OR} 3,5)$. Dando como resultado que un 64,7 \% de los 
animales seropositivos tuvieron problemas entéricos en los meses anteriores y un $65,4 \%$ de los animales negativos no los tuvieron.

Al evaluar el acceso a desechos orgánicos, se hallo una relación entre los animales positivos y el libre acceso a este tipo de desechos $(\mathrm{p}<0,05)(\mathrm{OR} 0,4)$. El estudio mostró que un $51,3 \%$ de los animales positivos permanecían sueltos, teniendo libre acceso a este tipo de desechos, entre tanto un 68,6 \% de los animales que resultaron negativos permanecían la mayor parte del tiempo dentro de los hogares, teniendo un acceso restringido a estos desechos.

En este estudio no se observó relación entre los siguientes factores y los animales seropositivo: Tipo de dieta $(\mathrm{p}>0,05)(\mathrm{OR} 0,6)$; el habitad y las condiciones higiénicas $(\mathrm{p}>0,05)(\mathrm{OR} 1)$; la convivencia con otros animales $(\mathrm{p}>0,05)(\mathrm{OR} 1,4)$; la desparasitación $(\mathrm{p}>0,05)(\mathrm{OR} 0,6)$; las fuentes de agua $(\mathrm{p}>0,05)(\mathrm{OR} 0,6)$; la disposición de los excrementos $(\mathrm{p}>0,05)(\mathrm{OR} 0,5) ; \mathrm{y}$ la salud ( $>0,05)($ OR 2$)$.

\section{DISCUSIÓN}

Es de una gran importancia el hallazgo de la alta prevalencia serológica a Salmonella enteritidis en la población canina de Tunja (41,7 \%), muy superior a las prevalencias reportadas en otros estudios,(18) con una alta concentración de anticuerpos en los animales muestreados, en promedio de 1: 1366.

Es interesante observar como los niveles de concentración de anticuerpos obtenidos a partir de la muestra para el control positivo (1:80), son más bajos que los obtenidos a partir de una infección natural (1:1280). Esto nos demuestra que una infección activa logra títulos muy altos de anticuerpos. La presentación de títulos de anticuerpos elevados (1:2 560) al analizar las muestras, sin evidencia sintomatológica demuestra la posible presencia de animales portadores asintomáticos, convirtiéndose en focos de alto riesgo para la propagación de la Salmonella enteritidis.

Este estudio demostró como existen determinadas áreas dentro de la ciudad con altos títulos de la enfermedad, es el caso de las zonas sur y centro siendo la primera la de más alta prevalencia. Además se encontró una marcada correlación entre la presentación de problemas entéricos (diarrea) en los meses anteriores y la manifestación de serorreactores. Mostrando un alto nivel de riesgo como factor diseminador de la enfermedad (OR 3,5). 
Así mismo el estudio determino que aunque hay relación entre la presentación de animales positivos y el libre acceso a desechos orgánicos, hay que considerar a este parámetro como un factor de bajo riesgo en la predisposición a la enfermedad (OR 0,4).

Otros factores estudiados como la convivencia con otros animales y el estado de salud mostraron que aunque no existe asociación entre estos y los animales serorreactores, si se debe tener en cuenta el grado de riesgo que mostraron especialmente la presentación de enfermedades concomitantes (OR 1,4 y OR 2 respectivamente). Estos resultados implican un alto nivel de riesgo para la población humana y animal de la ciudad de Tunja •

Agradecimientos. Al laboratorio de microbiología de la Facultad de Medicina Veterinaria y Zootecnia de la Universidad Nacional de Colombia y a los propietarios de caninos en la ciudad de Tunja por permitirnos realizar este estudio.

\section{REFERENCIAS}

1. Gil-Setas A, Ramos M. Salmonelosis no tifoidea en un área de salud de Navarra, España. Rev. Esp. Salud Publica. 2002; 76, (1): 49-56.

2. Baron S. Salmonella Medical Microbiology. 4th Edition. Galveston The University of Texas Medical Branch;1999.

3. Hackett T, Lappin MR. Prevalence of enteric pathogens in dogs of north-central Colorado. J. Am. Anim. Hosp. Assoc. Jan-Feb2003; 39 (1): 52-6.

4. Fukata T, Naito F, Yoshida N. Incidence of Salmonella infection in healthy dogs in Gifu prefecture, Japan. J. Vet. Med. Sci. Nov 2002; 64 (11): 1079-80.

5. Suarez CM, Mantilla RJ. Presencia de Salmonella serovariedad enteritidis en productos de origen avícola y sus repercusiones en salud pública. IATREIA 2000; 13:237-245.

6. Weese JS, Peregrine AS, Armstrong J. Occupational health and safety in small animal veterinary practice: Non parasitic zoonotic diseases. Canadian Veterinary Journal. 2002. August; 43(8): 631-636.

7. Carter GR. Guide to Infectious and Parasitic Diseases of Dogs and Cats. Ithaca, N.Y: International Veterinary Information Service (IVIS) [Internet]; 2001 July. Disponible en: http:// www.ivis.org/special-books/Carter/Carter7/chapter-Frm.asp?=1 Consultado: 12 de noviembre 2006.

8. Cherry B, Burns A, Jonson SG. Salmonella Typhimurium Outbreak Associated with Veterinary Clinic. Emergin Infectious Diseases Journal; December 2004; Vol. 10 (12): 2 249-2 251.

9. Patronek GJ, Beck AM. Glickman LT. Dynamics of dog and cat populations in a community. JAMVA.1997; 210:637-42.

10. Urtiaga M, De Pablo N, Zabala. A. Situación de las enfermedades de declaración obligatoria (E.D.O.S) en Navarra. Red Nacional de Vigilancia Epidemiológica. Enfermedades de Declaración Obligatoria (EDO); 1998.

11. Tengelsen J, Leslie A. Multidrug resistant Salmonella Typhimurium in four animal facilities. American Veterinary Medical Association; July 2001.

12. Reinberg S. Las tortugas mascotas presentan un riesgo de Salmonella para los niños [Internet]. HealthDay News / Dr Tango; July 2007. Disponible en: http://salud.latino.msn.com/ embarazoehijos/articlepage.aspx?cp-documentid=100166131Consultado: octubre del 2007. 
476 REVISTA DE SALUD PÚBLICA · Volumen 10 (3), Julio 2008

13. Larrieu E. Manual de Epidemiología y Salud Pública Veterinaria. En: Cátedra de Epidemiología y Salud Pública. Facultad de Ciencias Veterinarias. U.N. La Pampa; 2003. p 8-14.

14. FAO. Publicación. Zoonosis en los sistemas de producción animal de áreas urbanas y periurbanas en América Latina. Livestock Policy Discusión Paper №2; Marzo 2001.

15. Ortega P. La sobrepoblación canina: Un problema de repercusiones potenciales para la salud humana. Rev. Biomed 2001; 12:290-291.

16. Hohenhaus A. Blood Banking and Transfusion Medicine. In: Veterinary Internal Medicine. 1998; Fifth Edition Vol 1,79:348-350.

17. Morilla A, Bautista C. Prueba de microantiglobulina (MAG) para la detección de infecciones producidas por salmonellas del grupo D y paratifoideas. En: Manual de Inmunología. Editorial Diana. México; Septiembre 1986. p. 303-304.

18. Morse EV, Duncan MA, Estep DA, Riggs WA, Blackburn BO. Canine salmonellosis: A review and report of dog to child transmission of Salmonella enteritidis. Am J Public Health. 1976 January; 66(1): 82-83. 